\title{
THE INFLUENCE OF ANTICOAGULANTS ON FIBRIN NETWORK FORMATION
}

\author{
BY \\ KAREL REJSEK AND MIRKO KUBÍK* \\ From the Clinic of Industrial Medicine and the Fourth Medical Clinic, Charles University, Prague
}

(RECEIVED FOR PUblication, MAY, 1948)

In recent years great efforts have been made to identify the stages of the process of coagulation. The object of this study was to demonstrate the effect, if any, of certain anticoagulants on the production of the fibrin network in a thrombus.

\section{Technique}

In general the method described by Fonio and Schwenderer (1942) was used. Ten ml. of blood taken from a fasting patient with a dry syringe was divided equally and put into two paraffined test tubes which had been kept in a refrigerator. Thereafter the blood was centrifuged for 2 minutes at 3,000 revolutions per minute. The plasma thus obtained was transferred in a dry, carefully cleaned pipette to a slide which had been sprinkled lightly with particles of burned kaolin powder under observation by a dark-field microscope. The kaolin particles were added to provide crystallization centres and focusing points. The preparation was then covered with a polished coverslip and observed by means of an oil-immersion objective in which an iris diaphragm was incorporated.

Two test-tubes were used because it had been found that the second sample frequently clotted spontaneously during centrifuging, a behaviour which could possibly be explained by the presence of bubbles of froth formed in expressing the blood from the syringe ; this factor might well influence blood-clotting. The microscope was focused either on suspended thrombocytes or better on the particles of kaolin. The total time, from the drawing of blood until the focusing on the preparation, was approximately 7 minutes.

\section{Normal Findings}

During the next 7 minutes the transformation of fibrinogen into fibrin occurred. The first fibrin needle suddenly formed on a crystallization centre (thrombocyte or kaolin particle); subsequently other crystals grew from it. In a short time fibrin crystals appeared on the other centres as well; and within 5 minutes the whole field was covered with a fibrin network, single " needles" being of varying lengths and forming an irregular reticulum. The development of the fibrin network was photographed by a "Mifilmca Leitz" camera, the exposure being 20 seconds with $23^{\circ}$ Scheiner cinema film.

The fibrin network was observed for several hours, some of the specimens being examined for as long as $\mathbf{2 4}$ hours. No considerable changes in the network were detected. Only occasionally, after 1 to 6 hours, haemochronia appeared in the interstices, showing a lively Brownian movement. These haemochronia have been seen even in the plasma of patients who had fasted for more than 12 hours, and they are therefore most likely an expression of the normal lipaemia.

\section{Observations on Changes Produced by Anticoagulants}

In cases with a lowered prothrombin level produced by the coumarin compound "pelentan" (Kubík and Reiniš, 1948), a difference in fibrin formation was noted the intensity of which was proportional to the degree of the fall in prothrombin level. The onset of the formation of the first fibrin needles was not delayed in comparison with the normal, but the development of the fibrin network proceeded more slowly. Delicate, long fibrin crystals were formed, producing an "asthenic" form of the network. The picture observed within 5 minutes in normal plasma was not reached until after several hours. A similar picture has been seen in icteric plasma, in which the prothrombin level has been lowered by hepatic damage or by retention of bile.

The effect of heparin was also observed. If added to blood in vitro, this substance interferes with coagulation for an unlimited time; even after seven days fibrin needles could not be detected. One $\mathrm{ml}$. of heparin $(50 \mathrm{mg}$.) was given intra-

\footnotetext{
* Now at the Department of Clinical Pathology, University College Hospital, London.
} 
Fig. 1.-Normal plasma. First fibrin needles suddenly appearing on a crystallization centre. (Time approximately 7 minutes after bleeding.) (Magnification of all pictures : microscopic, $\times 750$; photographic, $\times 27$ \%)

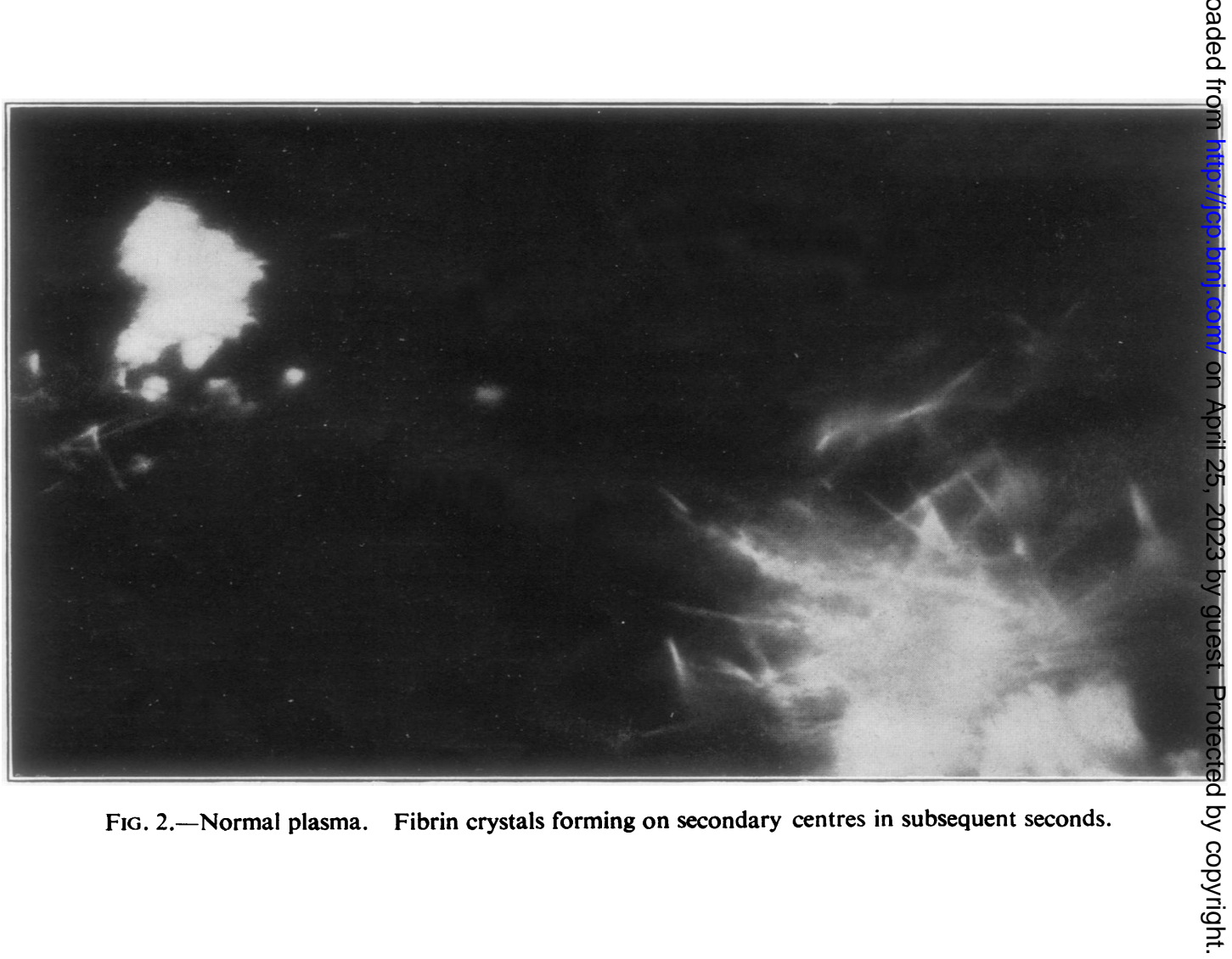




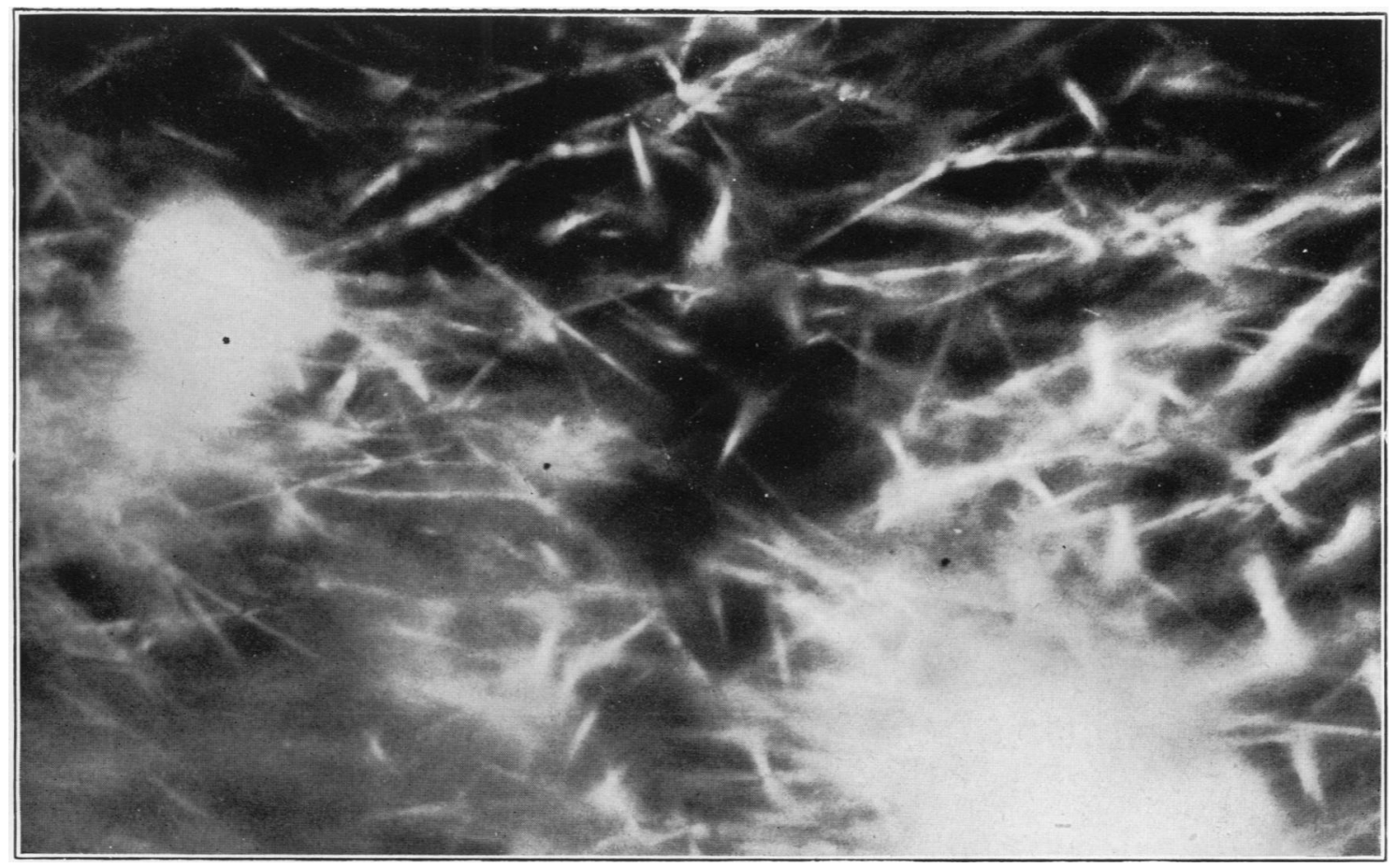

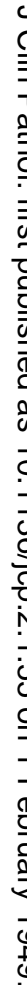

Fig. 3.-Normal plasma. Definite fibrin network made by large needles of varying lengths. (Time 5 to 7 minutes after formation of first crystals.)

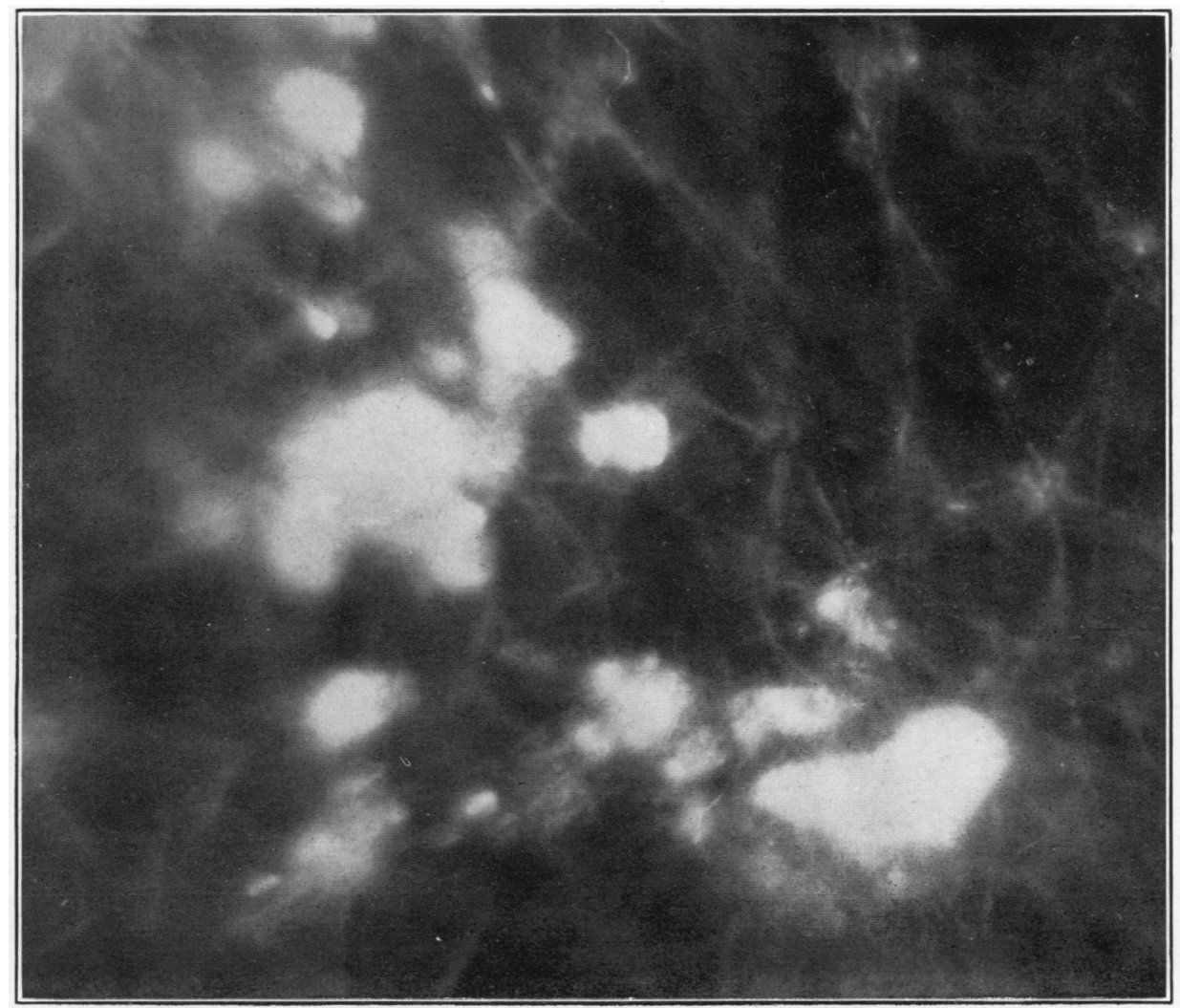

FIG. 4.-Plasma of a patient treated with a coumarin compound. Prothrombin level 25 per cent of normal. Only delicate, long fibrin crystals are seen, which developed after considerable delay. (Time 1 hour after bleeding.) 


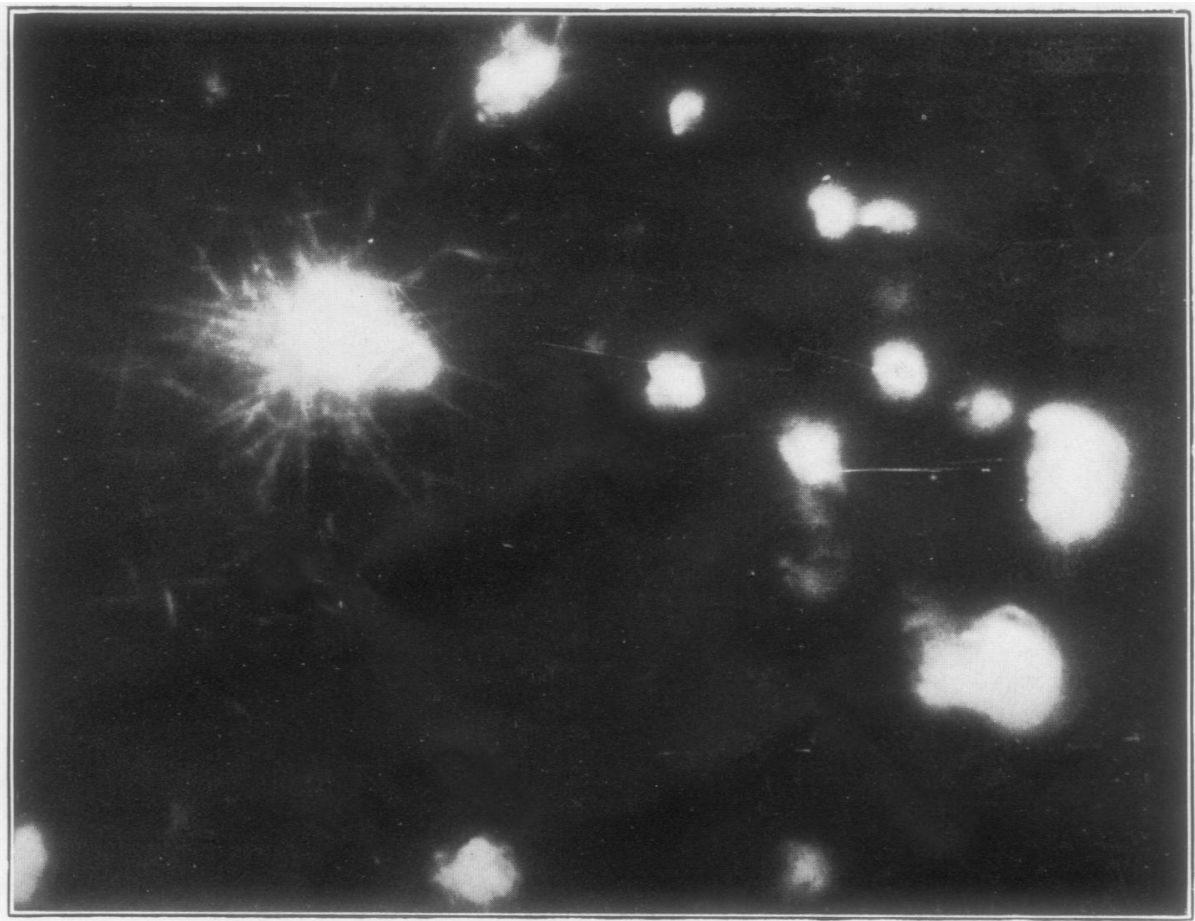

FIG. 5.-Plasma of a patient 3 hours after injection of $50 \mathrm{mg}$. of heparin. First phase of fibrin crystal formatio (Time 7 to 9 minutes after bleeding.)

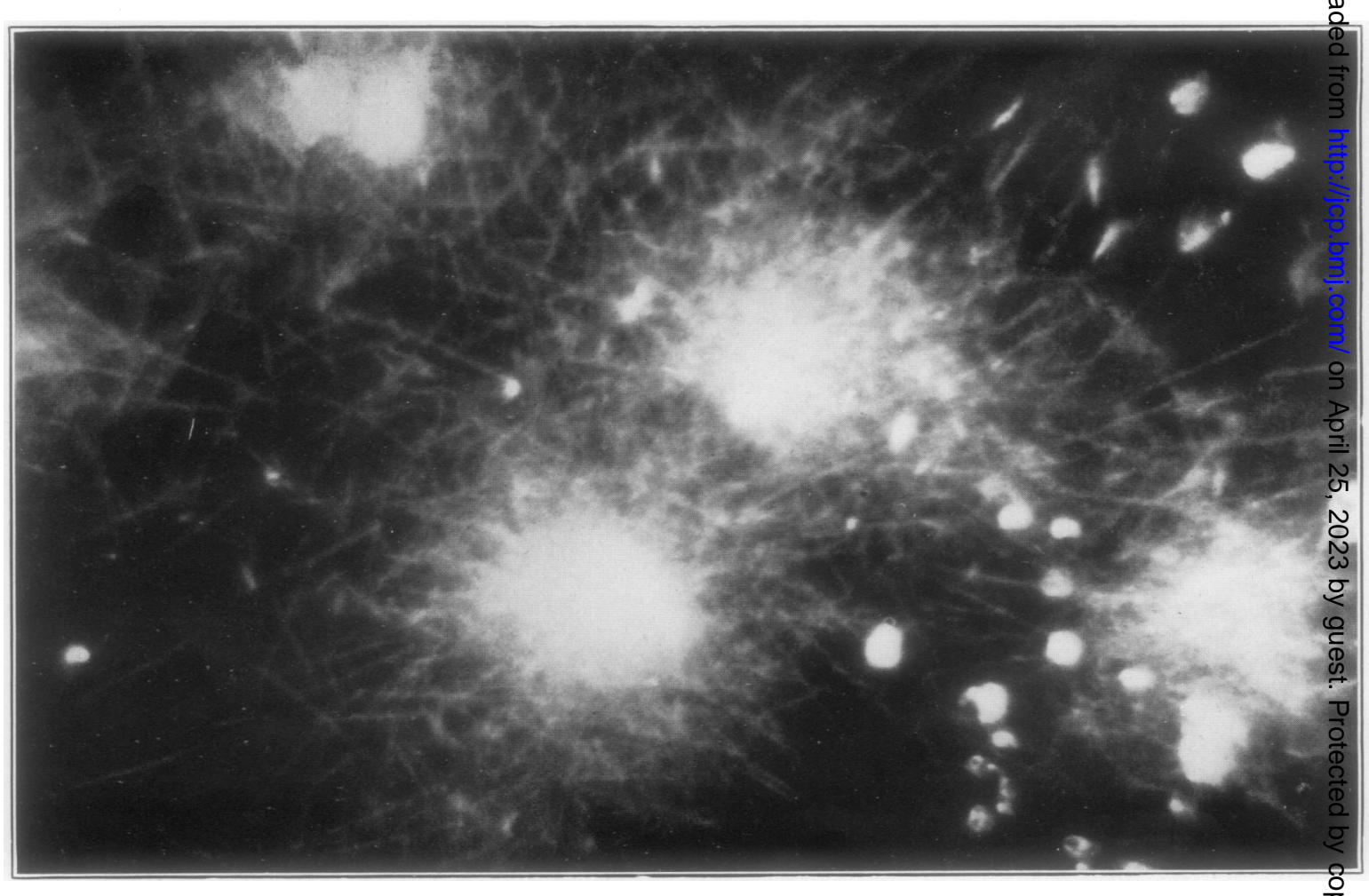

FiG. 6.-Plasma of a patient 3 hours after injection of $50 \mathrm{mg}$. of heparin. Delicate crystals heaped up aroum the crystallization points. Picture of definite network. (Time approximately 15 minutes after bleeding.) 
venously, and the first sample was taken 20 to 30 minutes after the injection. No crystals were seen to form in these specimens when they were examined immediately; the blood in the test-tube remained unclotted. Twenty-four hours later, however, if a specimen were taken from this testtube, the fibrin network formed within the normal time. It appears that passage through the body so alters heparin that its efficacy becomes exhausted in the blood in the test-tube. This late network has different morphological properties which are the same as those seen in blood taken 3 hours after a previous heparin injection. In both, the time of appearance of fibrin crystals formation is normal ( 7 minutes) after the slide has been prepared, or approximately 15 minutes after the blood has been withdrawn. Although the velocity of development of the network is apparently about the same as in normal plasma, the crystals are more delicate, and the mesh of the network is smaller, with characteristic " hedgehog" formations heaped up around the centres of crystallization.
Because it is extremely difficult to reproduce in vitro all the biochemical and mechanical factors which take part in this reaction, exact conclusions cannot be drawn. Some artificial conditions are produced in the preparation of the specimens which differ from intravascular conditions. Nevertheless, it is felt that in this way a survey was obtained of the morphology of this phase of the process of coagulation, and the findings provide some idea of the structure of a thrombus and how this is influenced by anticoagulants.

\section{Summary}

By means of a dark-field technique the formation of fibrin network has been observed. Findings under normal conditions, and changes caused by administration of heparin and of a coumarin compound, are described.

\section{REFERENCES}

Fonio, A., and Schwenderer, J. (1942). "Die Thrombozyten des menschlichen Blutes." Berne: B. Schwabe.

Kubík, M., and Reiniš, Z. (1948). Schweitz. med. Wschr., 78, 785. 


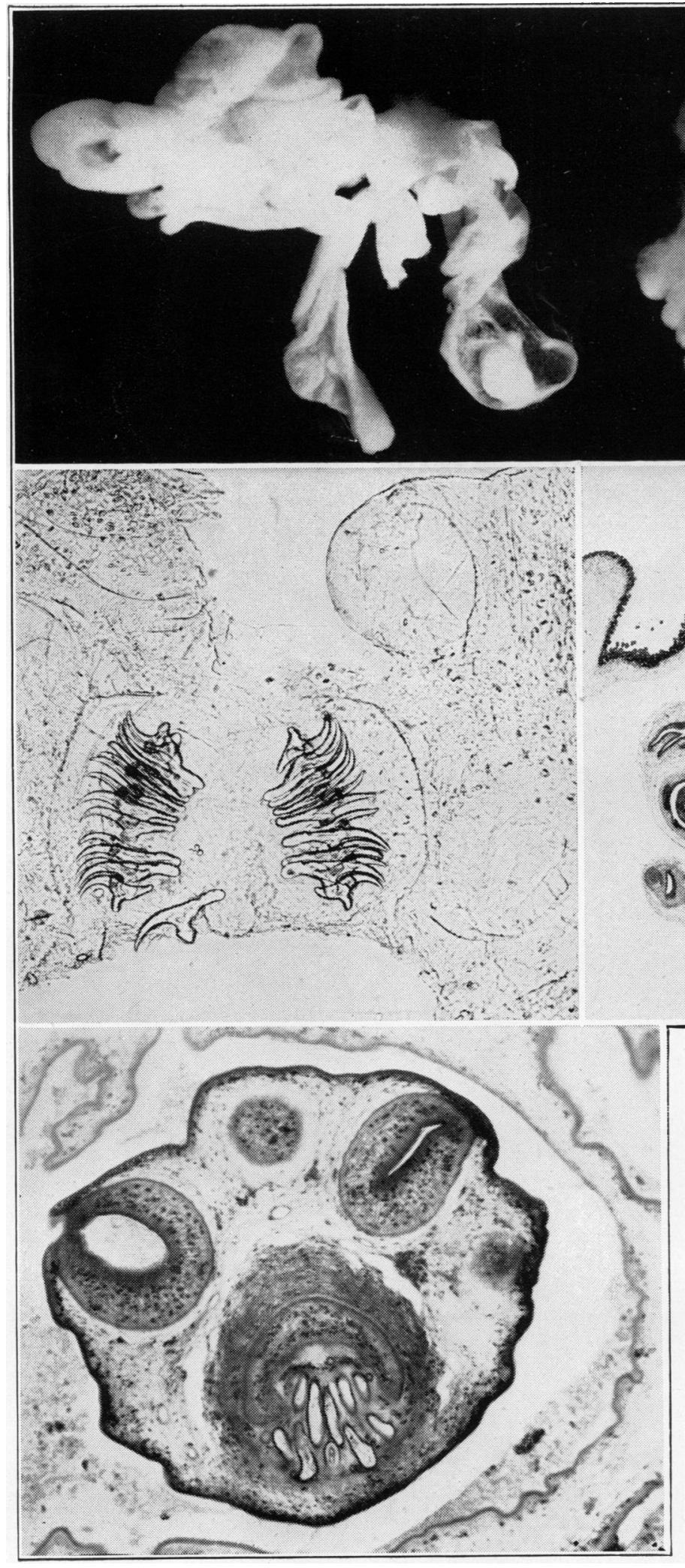

Above, left:

FIG. 1.-Portions음

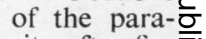
site after fix-क ation in form- $\vec{\Phi}$ a ld ehyde.으 (Scale 4.50 $\mathrm{mm}$. = 1 s inch.) $\vec{\circ}$ 